\title{
Erratum to: Inequalities in the frequency of free sugars intake among Syrian 1-year-old infants: a cross-sectional study
}

Easter Joury ${ }^{1,2^{*}}$, May Khairallah², Wael Sabbah ${ }^{1}$, Kanaan Elias ${ }^{3}$ and Raman Bedi ${ }^{4}$

\section{Erratum}

Unfortunately, the original version of this article [1] was missing funding information within the funding section.

Please note that open access for this article was funded by King's College London.

\footnotetext{
Author details

'Population and Patient Health, King's College London Dental Institute, Denmark Hill Campus, Bessemer Road, London SE5 9RS, UK. ${ }^{2}$ Oral Medicine Department, Faculty of Dentistry, Damascus University, Damascus, Syria.

${ }^{3}$ Eastman Dental Institute, University College London, London WC1X 8LD, UK.

${ }^{4}$ Centre for International Child Oral Health, King's College London, 26-29

Drury Lane, Rooms 329-331, London WC2B 5RL, UK.
}

Received: 8 September 2016 Accepted: 20 September 2016

Published online: 07 October 2016

\section{References}

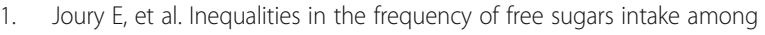

Syrian 1-year-old infants: a cross-sectional study. BMC Oral Health.

2016:16:94. doi:10.1186/s12903-016-0287-8.

\footnotetext{
* Correspondence: easter.joury@kcl.ac.uk

'Population and Patient Health, King's College London Dental Institute,

Denmark Hill Campus, Bessemer Road, London SE5 9RS, UK

${ }^{2}$ Oral Medicine Department, Faculty of Dentistry, Damascus University,

Damascus, Syria

Full list of author information is available at the end of the article
} 